\title{
Respiratory responses of diabetics to hypoxia, hypercapnia, and exercise
}

\author{
JG WILLIAMS, AI MORRIS, RC HAYTER, CM OGILVIE \\ From the Department of Medicine, University of Liverpool, and Walton Hospital and the Royal Liverpool \\ Hospital, Liverpool
}

ABSTRACT The respiratory responses of 52 diabetics and 65 non-diabetic controls to hypoxia, hypercapnia, and exercise were studied. Twenty five per cent of the diabetics had evidence of impaired sensitivity to hypoxia or decreased ventilatory response to hypercapnia, while $7 \%$ of the diabetics who performed the exercise tests had an abnormal pattern of respiration during exercise; $33 \%$ of the diabetics who performed all three tests of respiratory reflex action had at least one abnormal test response. There was no correlation between the presence of an abnormal respiratory response and the presence of clinical diabetic complications. Abnormal respiratory reflexes could not be predicted from the results of the "routine" pulmonary function tests. The possibility that the abnormal respiratory responses were due to autonomic neuropathy is discussed.

Autonomic neuropathy is being increasingly recognised as an important complication of diabetes. Disease of the cardiovascular system has been associated with a poor prognosis, ${ }^{\prime}$ and defective respiratory reflexes have been postulated as an aetiological factor in the causation of sudden and unexplained cardiorespiratory arrests in diabetics with symptoms of autonomic neuropathy. ${ }^{2}$

Despite an initial report, ${ }^{3}$ several subsequent studies have failed to demonstrate any impairment of the ventilatory response to hypoxia in diabetics with symptoms of autonomic neuropathy..$^{4-6}$ There are also conflicting reports on whether diabetics with peripheral neuropathy have an abnormally decreased response to hypercapnia. ${ }^{57}$ The present study was designed to assess the ventilatory responses of a randomly selected group of diabetics to hypoxia, hypercapnia, and exercise.

\section{Patients and methods}

THE SUBJECTS

There were 1202 patients, aged 16-65, attending the weekly diabetic clinic at Walton Hospital, Liver-

Address for reprint requests: Dr JG Williams, Walton Hospital, Liverpool L9 1AE.

Accepted 13 February 1984 pool, from May 1981 to February 1982 who were considered for inclusion in the study. Four hundred and eighty seven patients were excluded because they were suffering from a disease or receiving treatment that might have altered autonomic reflexes. Thus 715 diabetics were eligible for further study. Fifty two non-smoking diabetics who were clinically free from lung disease were selected by the random numbers method and performed the respiratory tests -30 men, mean age 33.6 years (SE $2 \cdot 42$ ) years, and 22 women, mean age $40 \cdot 3(3.60)$ years. Thirty five were receiving insulin treatment, eight were taking hypoglycaemic drugs, and nine were treated by diet alone. Sixty five non-diabetic subjects, all of whom were non-smokers and clinically free of lung disease, performed the respiratory tests $(30$ men, mean age $32.9(2 \cdot 1)$ years, and 35 women, mean age $32 \cdot 0(2 \cdot 27)$ years). Fifty of these subjects were recruited while they were attending the day ward at Walton Hospital for minor surgical procedures. The other 15 subjects were medical staff from the hospital. The mean height and weight of the control group were not significantly different from those of the diabetic patients.

A detailed history was obtained from all patients entering the study. Routine clinical examination was performed to detect the presence of peripheral neuropathy (absent tendon reflexes, impaired 
appreciation of vibration, touch, or pain) diabetic eye disease (cataracts and retinopathy), and peripheral arterial disease (absent pulses). Urine was checked for protein $(>0.3 \mathrm{~g} / 1)$.

All non-diabetic controls either had their urine checked for glycosuria or had a random blood sugar estimation performed to ensure that they were not symptomless diabetics, or had both tests done. The venous blood concentrations of potassium and sugar were estimated in 48 diabetics and the venous blood urea in 47 diabetics. The omissions were due to technical error or loss of sample. The glycosylated haemoglobin concentration was measured in 28 diabetics.

\section{RESPIRATORY TESTS}

Lung volumes and transfer factor

Static lung volumes and maximal breathing capacity were measured with the PK Morgan TLC-Test equipment.

The functional residual capacity was measured by the helium dilution technique. ${ }^{89}$ Dynamic lung volumes were measured with a bellows spirometer (Vitalograph). The single breath technique ${ }^{10}$ was used to determine the transfer factor for carbon monoxide, PK Morgan Model C transfer test equipment being used.

\section{Measurement of sensitivity to hypoxia}

A 6 litre anaesthetic bag was connected to a mouthpiece via a three way tap, which enabled the patient to breathe either air or the contents of the bag. The distal part of the mouthpiece was connected to a carbon dioxide analyser.

The subject was allowed to breathe air quietly for five minutes. He was then asked to exhale fully to residual volume at the end of a normal expiration so that an alveolar sample could be collected for measurement of carbon dioxide tension $\left(\mathrm{PCO}_{2}\right)$. The test procedure was started when three successive values for $\mathrm{PCO}_{2}$, measured at intervals of three to four minutes, were within $4 \mathrm{~mm} \mathrm{Hg}(0.53 \mathrm{kPa})$ of each other.

Subjects were asked to exhale to residual volume and then inhale to total lung capacity. Two different concentrations of oxygen were used $(10 \%$ and $100 \%$ ) and the order in which they were inhaled was decided by lottery. Breath holding time was measured by a stopwatch from the time the subject attained total lung capacity to the start of the next exhalation.

As volition is an important determinant of breath holding time, subjects were asked to perform this procedure for both concentrations of oxygen until the coefficient of variation for three successive readings was less than $10 \%$. This usually took four to six attempts.

The subjects' sensitivity to hypoxia was quantified by means of the following equation:

Breath holding time $(\mathrm{BHT})$ ratio $=\mathrm{BHT}_{100} / \mathrm{BHT}_{10}$, where $\mathrm{BHT}_{100}$ is the mean value of the breath holding time after a single breath of $100 \%$ oxygen and $\mathrm{BHT}_{10}$ is the mean value after a single breath of $10 \%$ oxygen.

\section{Measurement of ventilatory response to hypercapnia} The ventilatory response to hypercapnia in hyperoxic conditions was determined by Read's rebreathing technique." By the rebreathing of a hyperoxic gas mixture whose initial $\mathrm{PCO}_{2}$ is similar to the $\mathrm{PCO}_{2}$ of mixed venous blood an equilibrium is quickly established between mixed venous blood, arterial blood, and expired gas. Changes of end expired $\mathrm{PCO}_{2}$ then reflect the changes of $\mathrm{PCO}_{2}$ at the chemoreceptors. The tension of carbon dioxide is assumed to be the same in the end tidal gas as in the bag. The slope of the line relating ventilation to arterial or alveolar $\mathrm{PCO}_{2}$ provides a measure of the ventilatory response to hypercapnia.

\section{Measurement of ventilatory response to exercise}

The ventilatory response to submaximal exercise on a cycle ergometer was measured in the following manner. The exercise was performed on an electronically braked Godart cycle ergometer and the electrocardiogram monitored continously by telemetry. Respiratory measurements were made by the PK Morgan Exercise Modulator Test System. Subjects inspired room air and expired via a pneumotachograph into a 5 litre mixing chamber, at the distal end of which mixed expired gas was sampled and analysed for oxygen and carbon dioxide. Ventilation was measured by electrical integration of airflow. Only subjects aged less than 55 years were exercised. All had a 12 lead ECG before the experimental session to exclude overt ischaemic heart disease. They were instructed beforehand to stop exercise if they experienced any chest or limb pain or any other untoward feeling.

The exercise equipment was calibrated before each test. Subjects were asked to cycle at $60 \mathrm{rpm}$ at each work rate. They initially cycled against no external work load until a steady state of ventilation was reached and then the work rate was increased by 10 watts each minute to a maximum of 80 watts. Patients were asked to stop before reaching a work rate of $\mathbf{8 0}$ watts if any of the following occurred: $(a)$ exhaustion; (b) cardiac arrhythmias; (c) their heart rate rose above $85 \%$ of the maximum predicted value.

The ventilation $\left(V_{1.0}\right)$-that is, the minute ventilation at an oxygen uptake rate of $1.0 \mathrm{l} / \mathrm{min}$-was 
calculated for each subject. The tidal volume contribution to ventilation was expressed as a percentage of the predicted $\mathrm{VT}_{30}$ (that is, the tidal volume at minute ventilation of $30 \mathrm{l} / \mathrm{min}$ ). This was derived from the following formula ${ }^{12}$ :

$$
\mathrm{VT}_{30}=0.13 \mathrm{VC}+0.87 \text {, }
$$

where VC is the vital capacity in litres.

\section{Other tests of autonomic function}

All subjects performed five tests of cardiovascular autonomic function (pressor responses to prolonged handgrip and standing, heart rate responses to standing, Valsalva manoeuvre, and deep breathing).$^{13}$

The resting pupil diameters of 25 diabetic patients and 25 non-diabetic controls were measured in conditions of darkness by means of a flash photograph of the subjects' eyes taken after 30 seconds in the dark. This is a sensitive method of detecting defective autonomic innervation of the pupil. ${ }^{14}$

\section{STATISTICAL METHODS}

Test results were log transformed wherever possible if the distribution of values for any particular test was positively skewed. Differences between the mean values of different groups were tested by using the Student's $t$ test and the Mann-Whitney U test. The intraindividual coefficient of variation was calculated by means of the following formula:

Intraindividual coefficient of variation $=$ standard deviation of observations $\times 100$

mean value of observations

Statistical analyses were performed with the Statistical Package for the Social Sciences on an IBM computer. ${ }^{1516}$

\section{DEFINITION OF NORMAL AND ABNORMAL RESPONSES \\ Breath holding time ratio}

The normal range of values for the results of the BHT ratio was defined as those values lying within two standard deviations of the mean of the control group of the same sex. A value for the BHT ratio was considered abnormal if it was below the mean and outside this range. Logarithmic transformation of the data was performed so that the values obtained were normally distributed. The logarithmic value of the ratio exactly two standard deviations below the mean was calculated and converted back into non-logarithmic form. A BHT ratio less than 1.23 in women or less than 1.26 in men was abnormal when calculated in this way.

\section{Carbon dioxide response}

The measurement of the logarithmic value of the carbon dioxide slope was used to assess an individual's response to hypercapnia. The values obtained in female controls were dependent on the age of the individual and therefore the predicted value for each individual had to be calculated with this factor taken into account. The following regression equations were calculated for both sexes from the results of the control groups.

Women: $\log \mathrm{CO}_{2}$ slope $=0.2379-0.0072 x$

$\mathrm{RSD}=0 \cdot 2158$

Men: $\log \mathrm{CO}_{2}$ slope $=0 \cdot 0958-0 \cdot 0004 x$

$\mathrm{RSD}=0 \cdot 222$,

where $x=$ age in years and RSD $=$ residual standard deviation.

The lower limit of the normal range was defined as the 2.5 percentile. This value was obtained by subtracting twice the residual standard deviation of the distribution of the dependent variable (age) from the relevant regression equation.

\section{Exercise indices}

The normal ranges of values for the exercise indices were defined as those values lying within two standard deviations from the mean of the control group's values for individuals of the same sex. A value for $\mathrm{VT}_{30}$ was considered abnormal if it was outside this range, irrespective of whether it was greater or less than the mean value of the control group.

\section{REPEATABILITY OF RESPIRATORY RESPONSES}

The intraindividual coefficients of variation for all the respiratory responses were measured in control subjects (table). Short term repeatability was determined from measurements taken on two successive days. The long term repeatability was determined from measurements taken on two separate

Repeatability of respiratory responses (expressed as mean coefficients of variation with standard deviations in parentheses)

\begin{tabular}{|c|c|c|}
\hline & \multicolumn{2}{|c|}{ Intraindividual variation } \\
\hline & Short term & Long term \\
\hline BHT ratio & $6.82 \% \quad(5.95)$ & $\begin{array}{l}12 \cdot 86 \% \\
n=7\end{array}$ \\
\hline Carbon dioxide slope & $24 \cdot 60 \%(19 \cdot 20)$ & $14.56 \%(10.62)$ \\
\hline$\dot{\mathrm{V}} \mathrm{E}_{1 \cdot 0}$ & $\begin{array}{lll}n=02 \% & (2.39)\end{array}$ & $5.91 \% \quad(2.32)$ \\
\hline$V_{T_{30}}$ & $\begin{array}{c}n=6 \\
6 \cdot 12 \% \\
n=6\end{array}$ & $\begin{array}{l}n=0 \\
10 \cdot 04 \% \\
n=6\end{array}$ \\
\hline
\end{tabular}

BHT_breatholding time; $\dot{\mathrm{V}}_{\mathrm{E}_{1} .0-\text { minute ventilation at oxygen }}$ uptake of $1.0 \mathrm{l} / \mathrm{min} ; \mathrm{VT}_{30}$ - tidal volume at minute ventilation of $30 \mathrm{l} / \mathrm{min}$. 
occasions six to nine months apart. All estimations of the repeatability corresponded favourably with the results of other workers. ${ }^{17-24}$

\section{Results}

\section{PREVALENCE OF ABNORMALLY DECREASED} RESPONSES TO HYPOXIA AND HYPERCAPNIA The respiratory responses of 52 diabetics to hypoxia and hypercapnia were compared with the responses of 62 control subjects who performed the breath holding test and 63 control subjects who performed the test of hypercapnic response.

Twelve diabetics had a value for the BHT ratio below the lower limit of the normal range of values found in the control subjects (fig 1). Seven diabetics and one control subject had abnormally decreased values for the slope of the carbon dioxide response curve. The prevalence of an abnormal response to either hypoxia or hypercapnia was $25 \%$ in the diabetic and $1.5 \%$ in the control group. This was a significant difference $(p<0.001)$. Five diabetics had an abnormal response to both hypoxia and hypercapnia.

\section{PREVALENCE OF ABNORMAL RESPIRATORY RESPONSES TO EXERCISE}

Fifty four control subjects and 42 diabetics performed exercise tests of respiratory function. Three diabetics had values of $\mathrm{VT}_{30}$ which were greater than $135 \%$ of their predicted values. These were considered to be significantly abnormal results, as opposed to those values which were just outside the previously defined normal range (fig 2). Apart from one diabetic, all control subjects and diabetic patients had normal values for $\mathrm{VE}_{1 \cdot 0}$.

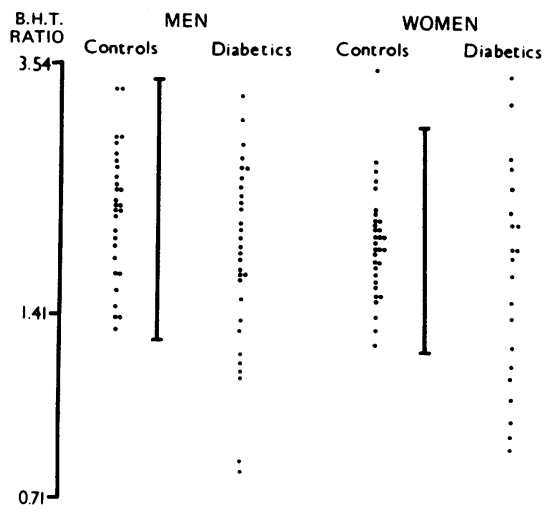

Fig 1 Distribution of values for the breath holding time (BHT) ratios in control subjects and diabetics. The bars represent 2 standard deviations from the mean for the control groups.

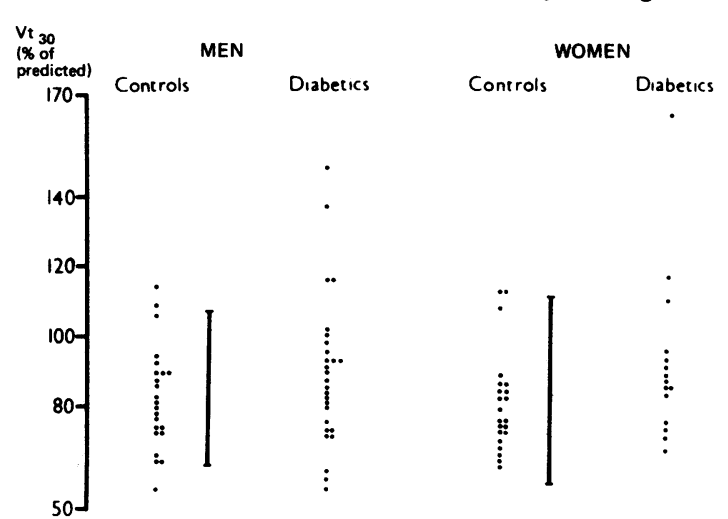

Fig 2 Distribution of values for tidal volume at minute ventilation of $30 \mathrm{l} / \mathrm{min}\left(V T_{30}\right)$, expressed as percentages of predicted values in control subjects and diabetics. The bars represent 2 standard deviations from the mean for the control groups.

\section{CONCURRENT ABNORMALITIES OF RESPIRATORY RESPONSES TO HYPOXIA, HYPERCAPNIA, AND EXERCISE}

Fifty one control subjects and 42 diabetics had undergone assessment of their ventilatory responses to all three stimuli-hypoxia, hypercapnia, and exercise. Seventeen diabetics $(33 \%)$ had at least one abnormal test result and five $(10 \%)$ had two abnormal results. Only one control subject had an abnormal result $(2.4 \%)$. The difference in the prevalence of one or more abnormal respiratory responses between the diabetic and control groups is significant $(p<0 \cdot 001)$.

\section{CLINICAL AND BIOCHEMICAL FINDINGS IN DIABETICS WITH AND WITHOUT ABNORMAL RESPONSES TO RESPIRATORY TESTS Diabetic condition}

The details of the diabetic condition in those diabetics with abnormal responses in respiratory tests were compared with those of the diabetics with normal responses. The duration of diabetes was not significantly longer in the diabetics with abnormal results than in those with normal results. There were no significant differences in the mean values for age or for concentrations of blood sugar, potassium, urea or glycosylated haemoglobin between the two groups.

There were also no differences in the distribution of the sexes, treatment, or prevalence of complications (that is, neuropathy, eye disease, and proteinuria) between the two groups of diabetics, the prevalence of the individual complications in the diabetic group as a whole being $25 \%, 22 \%$, and $4 \%$ respectively. 
Responses to respiratory function tests and other tests of autonomic function

Mean values for functional residual capacity, vital capacity, residual volume, total lung capacity, transfer factor, and maximal breathing capacity were not significantly different between the diabetics with and without abnormal respiratory responses.

There was no significant difference in the mean values of the cardiovascular responses between the diabetics with and without abnormal respiratory responses. Only one diabetic had evidence of abnormal autonomic reflexes in both systems.

Among the 25 diabetics whose autonomic innervation to the pupil was assessed, the mean value for the pupillary diameters of the nine diabetics with abnormal respiratory responses was significantly smaller than that of the 16 diabetics with normal respiratory responses $(5.55$ (SD 0.45$) \mathrm{mm} v 7.19$ $(0.28) \mathrm{mm}: \mathrm{p}<0.01)$. The mean pupil diameter of the control group was $6.08(0.22) \mathrm{mm}$.

\section{Discussion}

Although abnormal ventilatory responses to hypoxia have been described in familial dysautonomia, only one patient with diabetes has previously been found to have a similar abnormality. ${ }^{3}$ Measurements of the ventilatory response to hypoxia reflect the function of the afferent and efferent limbs of the reflex arc. The results are of limited value for detecting an abnormally low ventilatory response to hypoxia because of the large range of values for a normal response. This may explain why other workers have not been able to detect impaired ventilatory responses to hypoxia in diabetics. ${ }^{4-6}$

The breath holding time is related to lung volume and alveolar concentration of oxygen before breath holding. ${ }^{25}{ }^{26}$ Davidson $e t$ al $^{27}$ showed that the carotid bodies are important mediators of the effect of level of oxygenation on the breath holding time. They studied six normal subjects and five symptom free asthmatics who had undergone carotid body resection. The BHT was similar in the two groups after the inspiration of $100 \%$ oxygen as peripheral chemoreceptor activity is negligible at this concentration of oxygen. The breath holding time in the group with resected carotid body, however, was twice that of the control group when $12 \%$ oxygen was inhaled. Furthermore, there was no change in the level of ventilation in those who had had the carotid body resected when they were exposed to hypoxia for three minutes followed by $100 \%$ oxygen for one minute, confirming that the breath holding time was an indicator of peripheral chemoreceptor activity.
Possibly hypoxia influences BHT by a local effect on the diaphragmatic muscle, but Agostoni ${ }^{28}$ concluded that a hypoxic stimulus was not a major factor in triggering diaphragmatic activity.

We decided to measure the ratio of BHT at two different concentrations of oxygen in view of the known linear relationship between breath holding time and alveolar oxygen concentration. ${ }^{26}$ Its value is independent of the lung size of the individual as the two values for breath holding time are taken from the commencement of breath holding at identical lung volumes. Repeatability was good. We conclude that the BHT ratio is an index of chemoreceptor function and that the abnormal values in our 12 diabetics are due to a defect in the reflex pathways for the respiratory response to hypoxia.

Homma et al, ${ }^{7}$ using the technique of Read," reported that diabetics with peripheral neuropathy had reduced ventilatory responses to hypercapnia, but Soler and Eagleton ${ }^{5}$ failed to confirm this using a different technique. In the present study seven diabetics had an impaired response to hypercapnia, but only two of these had clinical signs of peripheral neuropathy. Loss of integrity of motor descending pathways to the respiratory muscles is a possible cause of a reduced ventilatory response to hypercapnia, but this is unlikely in our study as the maximal voluntary ventilation of these patients was similar to that of the diabetics with normal respiratory responses. A decreased ventilatory response to hypercapnia may occur as a result of a reduction in the amount of afferent information arising from the carotid bodies. ${ }^{29}$ The insensitivity to hypoxia shown by some of the diabetics in the present study suggests that the afferent pathways from the carotid bodies are affected in diabetes; this may also be the reason for the abnormally decreased ventilatory response to hypercapnia.

Three diabetics had values of $\mathrm{VT}_{30}$ which were greater than $135 \%$ of their predicted values. $\mathrm{VT}_{30}$ is greater in athletes than non-athletes but this is unlikely to be the reason for the large values that we have observed as none of our subjects was undergoing any type of training. Experimental evidence from dogs has shown that the contribution of tidal volume to minute ventilation during exercise increases after a vagotomy. ${ }^{30}$ The excessively large $\mathrm{VT}_{30}$ of the three diabetics with values greater than $135 \%$ may therefore have been due to vagal neuropathy.

There were no significant differences in the results of routine pulmonary function tests between the control subjects and diabetics or between diabetics with abnormal respiratory responses and those with normal respiratory responses. It follows that none of the measurements of lung volume or resting ventila- 
tion performed during the study can be used as indicators of automonic neuropathy affecting the respiratory system.

The lack of a close relationship between evidence of abnormal respiratory responses and abnormal cardiovascular responses or peripheral neuropathy may be due to the difference in the sensitivities of the methods used to detect the different forms of neuropathy. Those diabetics with abnormal responses to respiratory tests, however, did have evidence of defective autonomic innervation elsewhere as their pupil diameters were significantly smaller than those of non-diabetics, small pupils being the commonest sign of autonomic neuropathy affecting the pupils.

Tables showing absolute values of all measurements of breath holding time have been lodged with the Medical Editor and are available from the author.

We are indebted to the late Dr MB Macaulay and Dr SB Cohen for allowing us to study their diabetic patients, Mr C West and Mr MCK Tweedie for their help with the statistical analysis of the results, Dr P Dean and his staff for estimating the glycosylated haemoglobin concentrations, and Mrs B Peattie and her team for their skilled technical help.

\section{References}

' Ewing DJ, Campbell IW, Clarke BE. The natural history of diabetic autonomic neuropathy. $Q J \mathrm{Med}$ 1980;49:95-108.

2 Page MM, Watkins PJ. Cardio-respiratory arrest and diabetic autonomic neuropathy. Lancet 1978; i:14-6.

${ }^{3}$ Courtney-Evans RJ, Benson MK, Hughes DTD. Abnormal chemoreceptor response to hypoxia in patients. Br Med J 1971;i:530-1.

${ }^{4}$ Calverley PMA, Ewing DJ, Campbell IW, et al. Preservation of the hypoxic drive to breathing in diabetic autonomic neuropathy. Clin Sci 1982;63:17-22.

${ }^{5}$ Soler NG, Eagleton LE. Autonomic neuropathy and the ventilatory responses of diabetics to progressive hypoxaemia and hypercarbia. Diabetes 1982; 31:609-14.

${ }^{6}$ Ewing DJ, Campbell IW, Calverley PWA, Fraser DM, Clarke BF. Natural history of diabetic autonomic neuropathy. $Q J$ Med 1979;48:660-2.

${ }^{7}$ Homma I, Kageyoma S, Takesi N, Toniguchi I, Sakai T, Abé M. Chemosensitivity in patients with diabetic neuropathy. Clin Sci 1981;61:599-603.

${ }^{8}$ Meneely GR, Kaltreider NL. The volume of the lung determined by helium dilution. Description of the method and comparison with other procedures. J Clin Invest 1949;28:129-39.

${ }^{9}$ Weiner RS, Cooper P. Measurement of functional residual capacity. Am Rev Tuberc 1956;74:729-38.

${ }^{10}$ Ogilvie CM, Forster RE, Blakemore WS, Morton JW. A standardised breath-holding technique for the clinical measurement of the diffusing capacity of the lung for carbon dioxide. J Clin Invest 1957;36:1-17.

" Read DJC. A clinical method for assessing the ventilatory response to carbon dioxide. Aust Ann Med 1967;16:20-32.

12 Cotes JE. Rational exercise testing: the case for simple indices for submaximal exercise: ventilation, tidal volumes and cardiac frequency. Scand J Respir Dis 1971; suppl 77:123-7.

${ }^{13}$ Ewing DJ, Clarke BF. Diagnosis and management of diabetic autonomic neuropathy. $\mathrm{Br}$ Med J 1982; 285:916-8.

14 Hreidarsson AB. Pupil size in insulin dependent diabetes. Diabetes 1982;31:442-8.

${ }^{15}$ Nie NH, Hull CH, Jenkins JG, Steinbrenner K, Bent DH. SPSS-statistical package for the social sciences. 2nd ed. New York: McGraw-Hill, 1975.

${ }^{16}$ Hull CM, Nie NH. SPSS update 7-9. New procedures and facilities for releases 7-9. New York: McGrawHill, 1981.

${ }^{17}$ Patrick JM, Tutty J, Pearson SB. Propranolol and the ventilatory response to hypoxia and hypercapnia in normal man. Clin Sci Mol Med 1978;55:491-7.

${ }^{18}$ Hutchinson PF, Harrison RN. Effect of acute and chronic beta-blockade on carbon dioxide sensitivity in normal man. Thorax 1980;35:869-72.

${ }^{19}$ Irsigler GB. Carbon dioxide response lines in young adults: the limits of a normal response. Am Rev Respir Dis 1976;114:529-36.

${ }^{20}$ Edwards RHT, Miller GJ, Hearn CED, Cotes JE. Pulmonary function and exercise responses in relation to body composition and ethnic origin in Trinidadian males. Proc $R$ Soc 1972;181:407-20.

${ }^{21}$ Hirshman CA, McCullough RE, Weil JV. Normal values for hypoxic and hypercapnic ventilatory drives in man. J Appl Physiol 1975;38:1095-8.

${ }^{22}$ Rebuck AS, Kangoleb M, Pengelly LD, Campbell EJM. Correlation of ventilatory responses to hypoxia and hypercapnia. J Appl Physiol 1973;35:173-7.

${ }^{23}$ Miller FJ, Cotes JE, Hall AM, Salvosa CB, Ashworth A. Lung function and exercise performance of healthy Caribbean men and women of African ethnic origin. $Q J$ Exp Physiol 1972;57:325-41.

${ }^{24}$ Spiro SG. Exercise testing in clinical medicine. Br J Dis Chest 1977;71:145-72.

${ }^{25}$ Mithoefer JC. Breath-holding. In: Handbook of physiology. Section 3: Respiration. Vol 2. Washington DC: American Physiological Society, 1965:1011-23.

${ }^{26}$ Otis AB, Rahn H, Fenn WO. Alveolar gas changes during breath-holding. Am J Physiol 1948;152:674-86.

${ }^{27}$ Davidson JT, Whipp BJ, Wasserman L, Koyal SN, Lugliani R. Role of carotid bodies in breath-holding. $N$ Engl J Med 1974;290:819-22.

${ }^{28}$ Agostoni E. Diaphragm activity during breath-holding: factors related to its onset. $J$ Appl Physiol 1963; 18:30-6.

${ }^{29}$ Guz A, Noble MIM, Widdicombe JG, Trenchard D, Mushin WW. The effect of bilateral block of vagus and glossopharyngeal nerves on the ventilatory response to $\mathrm{CO}_{2}$ of conscious man. Respir Physiol 1966;1:206-10.

${ }^{30}$ Phillipson EA, Hickey RF, Bainton CF, Nadel JA. Effect of vagal blockade on regulation of breathing in conscious dogs. J Appl Physiol 1970;29:475-9. 